\title{
Permanent Resident Virus in Human Beings Viral Microecology of Human Being
}

\author{
Chen Guomin ${ }^{1}$ and Junru Wu $\mathbf{W}^{2^{*}}$ \\ ${ }^{1}$ Chongqing Medical University, China \\ ${ }^{2}$ Department of Physics, University of Vermont, USA
}

*Corresponding author: Junru Wu, Department of Physics, University of Vermont, USA.

To Cite This Article: Junru Wu. Permanent Resident Virus in Human Beings Viral Microecology of Human Being. Am J Biomed Sci \& Res. 2019 3(3). AJBSR.MS.ID.000667. DOI: 10.34297/AJBSR.2019.03.000667

Received: June 03, 2019 | Published: June 10, 2019

\section{Preface}

For analysis of the origin of life, viruses are compatible with cells. The formation, survival, and development of virus depend absolutely on cells. The existence of viruses must have their longterm host for survival, spreading and extending. Like other species in nature, human beings also coexist with a group of human-hosted viruses, which are called permanent resident viruses in human beings. The immune system of species has the anti-microbial invasion function. The virus has a special coexistence mechanism with its long-term host, who has a perfect immune system. Since the immune system has no capacity for entering into cells to recognize and remove viral nucleic acids, the invading viruses can reside in cells for a long time as a recessive existence under the dynamic balance between residual viral replication and immune clearance pressure. In this normal state, the virus and its long-term host are "stakeholders" living harmoniously. Consequently, the normal state of all natural viruses after the occurrence of the immune system of the species, constitutes the viral microecology of the host (excluded viruses parasitic air cavities in microorganisms).

Normal state viruses can undergo antigenic mutation to break through immune pressure and become abnormal, then viruses can proliferate again and spread to cause infection in host population. The abnormal state would return into normal state after a new group immune protection to be established again. The primary infection of the normal state virus and the re-infection of the abnormal state virus can cause disease due to the immune toxicity caused by the immune response. The identification of permanent resident viruses in human beings has important significance for studying the epidemiology, pathogenesis, clinical manifestations and outcomes of human viral diseases and epidemics, as well as disease prevention and treatment. Vaccine can successfully prevent the diseases caused by initial infection of normal state viruses. However, for the re-infection of abnormal state viruses, it is difficult to evaluate the preventive effect of vaccines because of the matching problem between previous vaccines and new antigens of virus in abnormal state.

\section{The Origin and Evolution of Permanent Resident} Viruses (PRVS) in Human Beings

\section{Endogenous virus with cell-origin}

Life can be considered as a material system in nature that can self-sustain, multiply and evolve [1-7]. The gene, from the occurrence in primitive marine vesicles to the appearance of cell life, is the most basic molecular system that can self-replicate and evolve in nature. It can be roughly divided into three developmental stages: gene, genome and cell genome. In the first two stages, various genes and randomly linked genomes develop equally, independently and competitively in vesicles. When the original vesicles evolve with the cell genome formation [2], those independent genomes developed in the same period are trapped in parasitic state and they either develop adaptively and to be able to sustainable or to be excluded in the process when cell genome gradually controls the whole cell performance, only those that are compatible with the cellular genome and the intracellular environment are retained. Some independent free genomes may shuttle between cells in the manner of cell division, proliferation, fusion, secretion, phagocytosis, sprouting, etc., Their structure and behavior already have the basic characteristics of virus, and become the endogenous virus of various primitive cells (endogenous virus). This is a very primitive virus and the source of all viruses in nature.

After entering the cell's life stage, the life cycle of original virus in the parasitic state can be completed by means of the genome function of the cell genome, therefore, the non-essential gene sequence [8] can be lost by nucleic acid replication "error", and the virus can develop along the "small but effective" direction for facilitating parasitism, so the viral genome has almost no static and inactive sequences [9]. The DNA genome is formed by linkage of 
gene sequences; while RNA cannot develop into a genome in the same manner due to molecular stability, but it can form an RNA genome by multiple RNA combinations through base pairing and second and third-order molecular configurations for completing complex biological functions, such as the ribosome genome does [10]. Due to stability limitations, the RNA genome contains a limited amounts of RNA, for example, influenza virus contains 8 RNA strands [11], while the reovirus genome consists of 12-14 RNA strands [12]. Therefore, the formation, structure, replication form and evolution of RNA virus [13] are completely different from those of DNA virus. These two types of viruses have no any relationship within the evolutionary mechanism.

\section{Permanent resident virus of multicellular higher species}

As the evolution of life entered the multicellular higher organism stage, the symbiotic endogenous virus also changes with cell differentiation and performance by corresponding adaptive changes, the endogenous virus then gradually evolved into the somatic cells of different tissues by compatibility selection and distribution. Therefore, different types of somatic cells may associate with their own endogenous virus population. The compatibility with the host cells determines the strict cytophilic characteristics of the virus, the hepatitis virus invades only liver cells, influenza virus invades respiratory epithelial cells, herpes viruses infect skin or mucosal epithelial cells, etc. With the development of germ cells and sexual reproduction mechanism in higher species, the virus continuation of such somatic cell has been greatly limited. Those endogenous viruses that have lost the transmission channel are eliminated.

The somatic virus developed from the endogenous virus can be long-term symbiotic with the host due to their compatibility. However, there is a significant difference in the transmission mechanism between the somatic virus and the viruses that are directly extended through reproductive passage. The somatic virus must evolve an external invasion process. Therefore, which is called the permanent resident virus (PRVs). For human being, due to the isolation of the reproductive mechanism of the placental barrier, in addition to the pseudo-viral sequences integrated into the genome during early evolution $[14,15]$, no existence of real endogenous viral particles has been confirmed.

\section{The trans- species infection and cyto-pathic virulence of} exogenous virus

Since the viral genome has the following characteristics:

a) Miniature size.

b) Lack of error correction mechanism for replication, it has the characteristics of fast copying and high replication error rate capacities.

Even in one host cell, the viruses can present in a group with various nucleic acid sequences, in other words, is in the form of quasi-species with a quasi-species spectrum [16]. The viral sequence analysis often represents the dominant strain with the highest detection probability. Certain base changes of sequence can lead to significant mutations in performance of the virus. When the surface (protein) antigens change by mutation, it might lead the virus to combine to the ligand or receptor of cells in another adjacent species. This invasion process is named trans-species infection [17], it means that of one PRVs of a species invades the cells of another species. Trans-species infection is an important mechanism for virus development and expansion of parasitic species, its occurrence depends on the probability of random variation of viral surface antigens, the contact density and genetic relationship in evolution between the two species [18]. For example, avian influenza virus, murine Hantavirus and canine rabies virus have invaded to infect human beings [19-21].

When the invasion of xenogeneic cells of trans-species, due to the lack of compatibility, there might lead the cell to suffer disease or even cause death by the interruption of cell metabolism with the process of virus replication and related substrates, this is defined as the viral cytotoxicity [22]. Cytotoxicity is a common feature of exogenous virus infection. The intensity of virulence depends on the route and quantity of virus invasion and the degree of incompatibility with cells. Because of incompatability, the hosts cannot carry viruses as a source of infection and lead to prevalence within the populations [23-25].

Because of the occurrence of viral cytotoxicity is based on incompatibility with cell level, in some case, this phenomenon can also occur between different types of somatic cells inside one body by trans-cells infection. This occurrence of cytotoxicity caused by viral mutations inside the body is more secretive. It can be applied to the pathogenesis of certain viral diseases, such as HIV concealed in macrophages infects CD4 cells by trans-cell infection resulting in AIDS [26-28], the cranial encephalitis caused by measles virus infecting brain nerve cells [29], these can cause clinical deterioration by cytotoxicity.

\section{Virus trans-species evolution and permanent resident virus (PRVs)}

Due to the characteristic of virus survival in quasi-species, the virus would gradually develop to quasi-species spectrum of various performance by replication [30], including virulence differences. With the survival advantage, the virus of the low virulence strain can survive relatively easier, and the virus quasi-species spectrum is gradually transferred to the low-virulence strain of adaptive in new host for continuation. This phenomenon is an important feature of virus survival, called "Passage attenuation of toxicity" [31]. Finally, the avirulent strain is eventually evolves into a subclass of homologous virus with a new species as a long-term host. Transspecies evolution is an important pathway and the universal law for virus development. For example, Flu virus through transspecies evolution, has been differentiated into various subtypes of influenza viruses that are prevalent in different species respectively for different hosts, such as human, poultry, pigs, and dogs [32,33]. At the same time, human influenza viruses also have various versions of viruses that have evolved from different sources. Transspecies evolution is a gradual process and of probabilistic nature [34]. If the compatibility of virus with host is too poor, they are difficult to coexist, the trans-evolution can be naturally terminated. 
For example, rabies virus and Hantavirus can infect human beings, however, there have no a subclass of viruses hosted by human beings for a long term. The HIV from the scorpion is a typical case. By the continuous subculture of the virus in a laboratory, the avirulent strain can finally be obtained which is one of the important ways to prepare the virus vaccine, and this also confirm the natural characteristics of passage attenuation of toxicity of virus.

Each subclass of one virus from trans-species evolution have their own long-term hosts. Due to the virus's the absolute dependence of on its host, each virus strains by successful evolved also lose the ability of reverting to infect the original species. Therefore, a virus in nature can only be prevalent in specific species, and there is no the same strain, which can be cross populated in both species simultaneously. In virology described, certain viruses can infect in a variety of species, as the Influenza virus epidemics can occur in human beings, poultry, pigs, dogs, etc. but it does not mean that the same virus strain (subclass) can spread to a large population in a variety of species.

The trans-species infection and evolution process of the virus occurs at the level of somatic cells of species, and it has the same invasive propagation characteristics as the PRV transformed from endogenous virus. Therefore, it is collectively named as PRV. Viral variation, trans-species infection, and attenuated evolution of passage are important mechanisms for studying viral sources and viral diseases. From the analysis of viral sequences, the vast majority of PRVs of human beings have genetic traces of similar viruses of adjacent species, showing that trans-species evolution of exogenous viruses is an important source of human's PRV populations [34] (Figure 1).

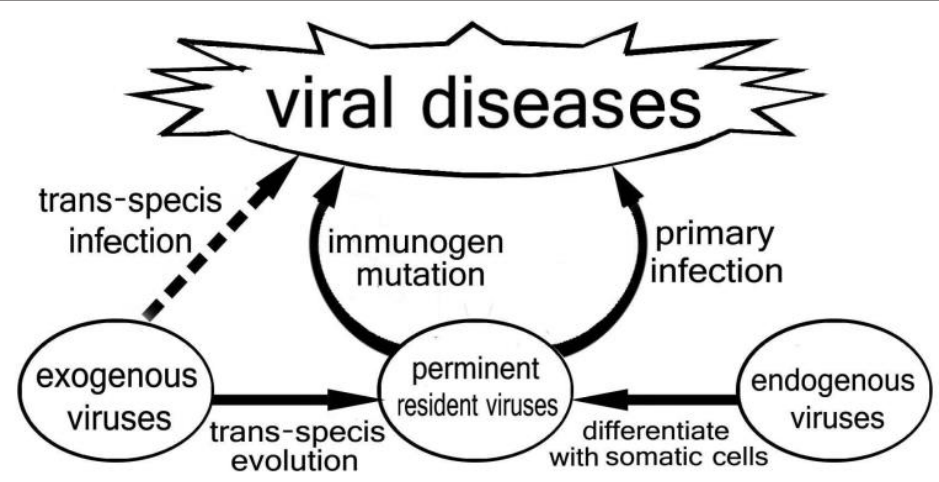

Figure 1: Evolution of the source of the resident virus population, viruses and diseases.

\section{Virus and immunity}

\section{PRV group and immune system}

The function of the immune system is to resist the invasion of microorganisms (including viruses) to maintain the body internal environment to be stable. The PRV can coexist with long-term host because it has no cytotoxicity inside the cells. However, the initial infection process can stimulate immune reaction of the host, then the effects of immune response can eliminate the viruses and infected cells, and provide immune protection against re-invasion and spread of similar viruses [35], Therefore, it must have to exist a special symbiotic mechanism for virus to survive in hosts with immune systems for a long term.

Immune function of multicellular organisms is evolved from those of the mutual recognition of cells based on cell surface markers, such as MHC antigens and Toll-like receptors [36,37], This recognition is based on the surface markers of the cell or microbial. Immune effector cells and molecules clear the virus or infected cells, and inhibit the virus to spread and develop through such molecular recognition of viral protein expressed on virus envelop or cell membrane. The virus exists as the form of nucleic acid in cells, and since the immune system has no mechanism to enter cells for recognizing nucleic acid sequence information and eliminating the nucleic acid sequence (viral genome), therefore, the resident virus can exist in the cell under the presence of the corresponding immune reaction, and replicate limitedly under immune-suppressive pressure. This condition of virus can maintain the stimulation to immune system for sustaining Immune stress. Finally, it would reach a dynamic balance between virus replication and immune clearance pressure. The presence of viruses in host under immune stress for relatively long term is generally the normal state of PRV group [1,38-40], and also involves the viral microecology of the host. It is also the normal state for all PRVs in their long-term hosts with immune system in the natural world [41-44]. The viruses of concealing survival could proliferate and escape as a source of infection from the individuals with even immune imbalance.

\section{Viral immune-toxicity and immune pathogenesis}

In the immune response cycle stimulated by the viral invasion, with the formation of immune complexes, cytolytic damage of infected cells, and the release and activation of various cytokines and inflammatory factors, it can lead to host diseases by local inflammatory injury and systemic inflammatory response, these immune-pathological phenomena in parallel to the immune response are called viral immune-toxicity[45]. The clinical manifestations are mainly caused by the immune-toxicity, this process is called immune-pathogenesis which is closely related to the immune response cycle $[46,47]$.

However, the immune-toxicity following immune response in the initial infection with PRVs is the main pathogenesis in host [45], so there exists a very regular incubation period of 8-10 days from 
the virus invading to clinical symptoms appearance [48]. During this period, the host becomes an asymptomatic source of virus as the virus can proliferate and spread freely in the body without immune clearance factors. When the clinical signs appearance in host, infection has already spread widely in advance leading to epidemics in population. The large-scale viral outbreak of viral diseases in human beings is mainly caused by PRVs of human. In addition to isolating the source of infection, the subsidence of the epidemic depends on the group's immune protection established by universal infection or vaccine application [49].

The clinical manifestations of PRVs infection depend mainly on the intensity of the immunopathology, the infection and immune process can be occult clinically. If the immune response is moderate in time, for example, there exist a huge recessive infection group behind the dominant group in the human flu epidemic, both of them get the immune protection after Flu. Otherwise it may manifest a very serious clinical process even causing disabling or lethal because the tissue damage is difficult to repair if the immune response is too strong or the host is extreme sensitive, such as the cases with severe smallpox, measles and polio cases.

Another important factor affecting clinical manifestations is the targeted cells or organs involved in immune-toxicity, which is directly related to the severity and the mortality of the disease. For example, the herpes virus invading the skin or mucous membranes rarely endangers human life and the human influenza virus invading the epithelial cells of airway have no direct lethal factor; however, the fatality rate of the Abola virus infection is high because the organ and systemic hemorrhage with vascular endothelial cells damage, and the morbidity rate of poliovirus that damage nerve cells are extremely high.

Immuno-toxicity is the main factor involved pathogenic mechanism of disease by PRVs. Clinical manifestations are caused by excessive immune response and body sensitivity. The appearance of immune-toxicity really indicates that the immune system has initiated the effects of clearing virus and infected cells, the severity of the disease is directly related the intensity of immune response. It is often necessary in clinical practice to use immunosuppressive therapy to control the intensive immune response into an appropriate level for sustaining life. Since the virus can survive under immunological stress after infection, there is no theoretical demand of enhancing the immune function to eliminate the virus.

\section{Virus and Disease}

\section{PRV and virus classification}

On the premise of understanding the occurrence, development, distribution, evolution of the virus and its absolute dependence on the cells, it is very clear to know that the virus must coexists with its long-term host, and its behavior is closely related to the hosts' life. In the long-term, the virus can spread widely within the population because of its compatibility to host sells and survives in an occult state under the control of the immune system. For example, we can speculate on the hidden existence of PRVs group from their corresponding antibodies in circulation of human beings. The occult virus may occur resurgence to escape from individuals due to events of immune imbalance happening (factors such as disease, aging, drugs, etc.). However, it is difficult to perceive these PRVs' existence clinically, due to it would not cause any abnormalities under the existing group immune protection.

It is known that one PRV species is really an exogenous virus for another species, and that when a PRV variant of a species invades other species by trans-species infection can directly lead the host to suffer a disease with cytotoxicity. However, it would not result in the epidemic in population because of the host can't bring the virus as a carrier to spread. For example, in the case of avian flu, the virus can escape accidentally to infect human beings, but it would not lead to the epidemic in human because lack of interpersonal transmission. In same way, the rabies virus also will not cause interpersonal communication. Therefore, for the studies of clinical virology the hosts must be clearly addressed. Understanding and identifying the PRVs of human beings for the study of human viral diseases and the prevention and treatment of epidemics are of great significance. For the viral diseases in human beings, concerned viruses can be divided into two categories: PRV group and exogenous virus group; They are totally different with infection mechanism, epidemic characteristics, pathogenic mechanism, clinical manifestations and outcomes, and prevention measures.

\section{PRV population and human viral epidemic}

The PRV group of human hosts has the close relationship to human beings with its affinity and compatibility to human cells, and the ability of interpersonal transmission in humans, so it is to be the main pathogen of human viral epidemics with widespread infection in the population. For the newborns, primary infection resulting to disease caused by immune toxicity is difficult to avoid. Therefore, it is clinically common for newborns with an average of 5-6 overt viral infections and a greater number of latent recessive infections per year [50]. The applications of vaccines have played a decisive role in preventing diseases caused by these initial infection of PRVs, these common viral diseases such as smallpox, measles and polio have almost disappeared in human beings [51]. Under the application of infant immunization programs in various countries, these diseases of primary infectious with PRVs have basically been controlled.

Except for primary infections, the normal state of PRVs under immune pressure could become into abnormal state due to antigen mutation to escape from existing immune pressure, then the virus can proliferate and spread again as a new PRVs due to the original group immune protection no longer effective. New viral antigens trigger the new immune responses leading to a new viral disease epidemic in the population, and eventually it would calm down after a new immune response creating a new equilibrium between virus and immune protective pressure. For example, the periodicity of the human influenza epidemic, the subsidence of the epidemic depends on the reconstruction of population immune protection. Theory and practice have shown that the viral mutations that lead to spread of such abnormal viruses and epidemic are occurring in human populations. The source of viral epidemic still originates from the antigenic variation of PRVs in population of humans themselves. 
The PRVs is the main virus group causing human viral epidemics. The disease is based on the immune pathogenesis, so the related diseases have regular disease processes and epidemiological characteristics. The pathogenesis of exogenous virus infection is both cytotoxic and immunotoxic, the clinical course is more complex, such as avian influenza virus, rabies virus, Hantavirus infection of humans, etc. Therefore, these two types of viral infectious diseases are easily identified clinically. Currently these epidemic viral diseases common in humans are all caused by viruses attributable to PRVs of humans, such as measles, smallpox, poliomyelitis, chickenpox, rubella, herpes virus, mumps, Japanese encephalitis, cytomegalovirus, hepatitis A, hepatitis B, dengue virus and human influenza virus, etc. including the HIV and HPV. According to the prevalence and pathogenesis, it is well known that SARS (Coronavirus), Ebola, MERS, and Zika should also belong to PRVs group of humans. These viruses have a common pathogenic mechanism, and the differences in clinical manifestations of various diseases are caused only by differences in invasion pathways and infected cells, the intensity of host immune response, and the difference in immune-pathological distribution in bodies. For example, the herpes virus invading the skin and epithelial cells is clinically mild, while the Ebola virus, which invades vascular epithelial cells, is critically ill and has a high mortality rate.

Due to the mechanism of passage attenuation, there is no cytotoxic variation in the long-term host virus. However, the PRVs viruses can cause trans-cells infections inside the body due to mutations, resulting in cytotoxicity and disease, such pathogenesis with AIDS [26-28] and measles encephalitis [29] as mentioned above. This pathogenic mechanism of PRV stems from viral cytotoxicity and does not involve immune pathogenesis, so the clinical manifestations are more subtle and complicated. Antiviral drugs are the main treatment in these special chronic viral infectious diseases such as AIDS.

\section{Prevention and treatment of human PRVs viral diseases}

The present clinical symptoms of these diseases indicate that the body has already initiated the immune response. The immunetoxicity is directly related to the intensity of the immune response. In addition to symptomatic treatment and overall support, the intensity of the immune response needs to properly be controlled to reduce the degree of injury in disease process, and finally achieve the balance of residues virus and immune pressure for rehabilitation. It is not advisable to enhance the immune response. Clinically, it is appropriate to use adrenocortical hormone to suppress an excessive immune response to save lives, as seen in the clinical treatment of SARS or Ebola.

The re-ignition of PRVs in the body due to the decline of immune function of the old, weak, and sick patients, is also an endogenous factor in the clinical viral infection of some critically ill patients (such as AIDS). Correctly elucidating the relative relationship between PRVs group and immunity has great theoretical significance for understanding the occurrence and prevention of viral diseases. Normal newborns can produce a normal immune response to foreign antigens, indicating that the immune systems of the newborns have matured since birth. It has been confirmed that a good immune response has been made for the hepatitis $B$ vaccine applied on the first day of human birth. The common viral infections in children are mainly caused by PRVs in humans. This is a learning process of immune system to obtain protection and difficult to omit for every individual. The common viral infections in children rarely occur in adults, it is contribute to the establishment of viral micro-ecology in the host after natural infection of PRV population or the application of corresponding vaccines.

\section{Vaccine and PRVs group in Humans}

The function of the vaccine is to simulate natural viral infections with artificial preparations, for establishing and maintaining corresponding immune protection under the premise of avoiding dominant immune-toxicity, this is the prevention of infectious diseases associated with immune pathogenesis. The attack on humans by those of exogenous virus group is opportunistic, so the application of the corresponding vaccine is selective. The PRVs of human is the main virus group that causes widespread infection in the population. Application of the vaccines has disappeared from smallpox and basically controlled the occurrence of common neonatal infectious diseases such as measles and polio. However, it is known that the PRVs can still residue in the cell in the presence of correspondent immune-protection. It is also well known that the vaccine is to eliminates the related disease but not virus, and the PRVs or live vaccine virus are still occult to present in the population [52]. The elimination of human variola virus is essentially replaced by vaccinia virus [53]. Newborn individuals still have the problem of primary infection of PRVs, so the immunization program of infants and young children needs to be maintained for a long time and can't be done once and for all $[54,55]$.

More importantly, the PRVs group has been existing in two states of normality and abnormality, and the vaccine plays a decisive role in preventing diseases caused by the primary infection of such normal state of viruses [51]. However, the preventive effect of vaccine on the epidemic of adult viral diseases is not ideal such as on those of influenza, because this kind of diseases in adults is caused by new immune-toxicity of the mutant virus in an abnormal state with breaking through the previous immune protection, its immunogen is different from those of vaccine applied in immune program for infant and young children. In theory, every episode induced by an abnormal state of PRVs requires the revalidation and verification of immunogenicity for vaccine, such as influenza vaccine. The theory and technology of SARS and Abola vaccine development in adults also need to be further explored [56,57]. At present, the prevalence of these viral diseases is naturally subsided without vaccines. The isolation of infectious sources plays an important role, then the technology of vaccine development and effects of vaccine applications for abnormal state of PRVs are yet to be evaluated in clinical practice.

\section{References}

1. Brockman MA, DM Knipe (2002) Herpes simplex virus vectors elicit durable immune responses in the presence of preexisting host immunity. J Virol 76(8): 3678-3687.

2. Chen G, J Wu (2017) The New Trend of Human Virology. International Journal of Bioanalysis \& Biomedicine 1(1): 1-9. 
3. Koblosh N (2015) [Features Microecology Genital Tract in Women Of Reproductive Age With Benign Cervical Pathology]. Lik Sprava 7(8): 98104.

4. Akram M, Tahir IM, Shah SMA, Mahmood Z, Altaf A, et al. (2018) Antiviral potential of medicinal plants against HIV, HSV, influenza, hepatitis, and coxsackievirus: A systematic review. Phytother Res 32(5): 811-822.

5. Greenwood B (2014) The contribution of vaccination to global health: past, present and future. Philos Trans R Soc Lond B Biol Sci 369(1645): 20130433.

6. Henderson DA (2011) The eradication of smallpox--an overview of the past, present, and future. Vaccine 29(4): D7-D9.

7. Lincoln T, G Joyce (2009) Self-sustained replication of an RNA enzyme. Science 323(5918): 1229-1232.

8. Tazzyman SJ, S Bonhoeffer (2015) BasicHousekeeping2Data from: Why there are no essential genes on plasmids. Revue Française De Sociologie 41(4): 777-796.

9. Mizokami M, Orito E, Ohba K, Ikeo K, Lau JY, et al. (1997) Constrained evolution with respect to gene overlap of hepatitis B virus. J Mol Evol 44(1): S83-S90.

10. Crick FHC (1968) The origin of the genetic code. J Mol Biol 38(3): 367379.

11. Butler D (2015) Flu genomes trace H7N9 evolution. Nature 519(7542): 137.

12. Flowers EM, Bachvaroff TR, Warg JV, Neill JD, Killian ML et al. (2016) Genome Sequence Analysis of CsRV1: A Pathogenic Reovirus that Infects the Blue Crab Callinectes sapidus Across Its Trans-Hemispheric Range. Front Microbiol 7: 126

13. Flores R, Selma Gago-Zachert, Pedro Serra, Rafael Sanjuán, Santiago F Elena (2014) Viroids: survivors from the RNA world? Annu. Rev Microbiol 68: 395-414.

14. Nowrouzi A, Glimm H, von Kalle C, Schmidt M (2011) Retroviral vectors: post entry events and genomic alterations. Viruses 3(5): 429-455.

15. Hughes JF, JM Coffin (2001) Evidence for genomic rearrangements mediated by human endogenous retroviruses during primate evolution. Nat Genet 29(4): 487-489.

16. Domingo E (2002) Quasispecies Theory in Virology. Journal of Virology 76(1): 463-465.

17. Gerald BP, Jeffrey BL, LM Wetzler (2004) Immunology, Infection, and Immunity. ASM PRESS Washington pp. 453-467.

18. Wágnerová M, Chalupková A, Hrabovská Z, Ančicová L, Mistríková J (2015) Possible role of different animal species in maintenance and spread of murine gamma herpesvirus 68 in the nature. Acta Virol 59(1): 14-9.

19. Velumani S, Du Q, Fenner BJ, Prabakaran M, Wee LC, et al. (2008) Development of an antigen-capture ELISA for detection of H7 subtype avian influenza from experimentally infected chickens. J Virol Methods 147(2): 219-225.

20. Hooper J, CS Schmaljohn (2002) Hantavirus. Springer Index of Viruses pp. 150-156.

21. Song H, Qi J, Xiao H, Bi Y, Zhang W, et al. (2017) Avian-to-Human Receptor-Binding Adaptation by Influenza A Virus Hemagglutinin H4. Cell Rep 20(5): 1201-1214.

22. Mahnel H (1968) Cytotoxicity of Virus. Cytotoxicity of Virus.

23. Ewald SJ, Kapczynski DR, Livant EJ, Suarez DL, Ralph J, et al. (2011) Association of Mx1 Asn631 variant alleles with reductions in morbidity, early mortality, viral shedding, and cytokine responses in chickens infected with a highly pathogenic avian influenza virus. Immunogenetics 63(6): 363-375.

24. CC (2010) Human rabies-Virginia, 2009. MMWR Morb Mortal Wkly Rep 59(38): 1236-1238.
25. Altun DU, IMA Öktem, B Ünal (2011) Hantavirus Infections and Prevention of Hantaviruses. Taf Preventive Medicine Bulletin 10(3): 373-378.

26. Sterjovski J, Melissa J Churchill, Anne Ellett, Lachlan R Gray, Michael J Roche, et al. (2007) Asn 362 in gp120 contributes to enhanced fusogenicity by CCR5-restricted HIV-1 envelope glycoprotein variants from patients with AIDS. Retrovirology 4: 89.

27. Xiao X, Wu L, Stantchev TS, Feng YR, Ugolini S, et al. (1999) Constitutive cell surface association between CD4 and CCR5. Proc Natl Acad Sci USA 96(13): 7496-7501.

28. Endres MJ, Clapham PR, Marsh M, Ahuja M, Turner JD, et al. (1996) CD4Independent Infection by HIV-2 Is Mediated by Fusin/CXCR4. Cell 87(4): 745-756.

29. Wisniewski HM, HR Brown, H Thormar (1983) Pathogenesis of viral encephalitis: Demonstration of viral antigen(s) in the brain endothelium. Acta Neuropathologica 60(1-2): 107-112.

30.Sanz-Ramos M, T Rodríguez Calvo, N Sevilla (2012) MutagenesisMediated Decrease of Pathogenicity as a Feature of the Mutant Spectrum of a Viral Population. PLOS ONE 7(6): e39941.

31. Lee C, August A, Arnold JJ, Cameron CE, et al. (2016) Polymerase Mechanism-Based Method of Viral Attenuation. Methods Mol Biol 1349: 83-104.

32. Ma S, K Downard, J Wong (2015) FluClass: A novel algorithm and approach to score and visualize the phylogeny of the influenza virus using mass spectrometry. Anal Chim Acta 895: 54-61.

33. Li Q, Wang X, Sun Z, Hu J, Gao Z, et al. (2015) Adaptive mutations in PB2 gene contribute to the high virulence of a natural reassortant H5N2 avian influenza virus in mice. Virus Res 210: 255-263.

34. Eigen M (1993) The origin of genetic information: viruses as models. Gene 135(1-2): 37-47.

35. Barker E (2004) Immunity to Virus. Immunology, Infection, and Immunity. Gerald B Pier (ASM Press. Washington D.C.): 463-467.

36. Carbone E, Terrazzano G, Ruggiero G, Zanzi D, Ottaiano A, et al. (1999) Recognition of autologous dendritic cells by human NK cells. Eur J Immunol 29(12): 4022-4029.

37. Jin YK, JO Lee (2011) Structural biology of the Toll-like receptor family. Annual Review of Biochemistry. 80: 917-941.

38. Xu L, Wei Y, Chen T, Lu J, Zhu CL, et al. (2010) Occult HBV infection in anti-HBs-positive young adults after neonatal HB vaccination. Vaccine 28(37): 5986-5992.

39.Zerbini A, Pilli M, Boni C, Fisicaro P, Penna A, et al. (2008) The characteristics of the cell-mediated immune response identify different profiles of occult hepatitis B virus infection. Gastroenterology 134(5): 1470-1481.

40. Lellouch Tubiana A, Fohlen M, Robain O, Rozenberg F (2000) Immunocytochemical characterization of long-term persistent immune activation in human brain after herpes simplex encephalitis. Neuropathology and Applied Neurobiology 26(3): 285-294.

41. Bandyopadhyay AS, Julie Garon, Katherine Seib, Walter A Orenstein (2015) Polio vaccination: past, present and future. Future Microbiol 10(5): 791-808.

42. Okayasu H, Sein C, Hamidi A, Bakker WA, Sutter RW (2016) Development of inactivated poliovirus vaccine from Sabin strains: A progress report. Biologicals 44(6): 581-587.

43. Abe S, Ota Y, Koike S, Kurata T, Horie H, et al. (1995) Neurovirulence Test for Oral Live Poliovaccines Using Poliovirus-Sensitive Transgenic Mice. Virology 206(2): 1075-1083.

44. Isanaka S, et al. (2017) Efficacy of a Low-Cost, Heat-Stable Oral Rotavirus Vaccine in Niger. N Engl J Med 376(12): 1121-1130.

45. Slon Campos J, J Mongkolsapaya, G Screaton (2018) The immune response against flaviviruses. Nat Immunol 19(11): 1189-1198. 
46. Moriyama T, Guilhot S, Klopchin K, Moss B, Pinkert CA, et al. (1990) Immunobiology and pathogenesis of hepatocellular injury in hepatitis B virus transgenic mice. Science 248(4953): 361-364.

47. Chisari FV (1996) Hepatitis B Virus Transgenic Mice: Models of Viral Immunobiology and Pathogenesis Curr Top Microbiol Immunol 206 149-173.

48. Hermesh T, Moltedo B, López CB, Moran TM (2010) Buying time-the immune system determinants of the incubation period to respiratory viruses. Viruses 2(11): 2541-2558.

49. Plans Rubio P (2012) Evaluation of the establishment of herd immunity in the population by means of serological surveys and vaccination coverage. Hum Vaccin Immunother 8(2): 184-188.

50. Gerald BP, Jeffrey BL (2004) Immunology, Infection, and Immunity. pp. 453-467.

51. Greenwood B (2014) The contribution of vaccination to global health: past, present and future. Philos Trans R Soc Lond B Biol Sci 369(1645): 20130433.

52. Abad C, N Safdar (2015) The Reemergence of Measles. Curr Infect Dis Rep 17(12): 51 .
53. Greenberg RN, JS Kennedy (2008) ACAM2000: a newly licensed cell culture-based live vaccinia smallpox vaccine. Expert Opin Investig Drugs 17(4): 555-564.

54. Morgan OW (2004) Following in the footsteps of smallpox: can we achieve the global eradication of measles? BMC Int Health Hum Rights 4(1): 1 .

55. Taniuchi M, Famulare M, Zaman K, Uddin MJ, Upfill-Brown AM, et al. (2017) Community transmission of type 2 poliovirus after cessation of trivalent oral polio vaccine in Bangladesh: an open-label clusterrandomised trial and modelling study. Lancet Infect Dis 17(10): 10691079.

56. Berger A, Drosten Ch, Doerr HW, Stürmer M, Preiser W (2004) Severe acute respiratory syndrome (SARS)--paradigm of an emerging viral infection. J Clin Virol 29(1): 13-22.

57. Sharma R, K Jangid, Anuradha, (2017) Ebola Vaccine: How Far are we? J Clin Diagn Res 11(5): DE01-DE04. 